\title{
Transient absorption of vibrationally excited ice $\mathbf{l} \boldsymbol{h}$
}

\author{
Adriaan M. Dokter ${ }^{a)}$ and Huib J. Bakker \\ FOM Institute for Atomic and Molecular Physics, Kruislaan 407, 1098 SJ Amsterdam, The Netherlands
}

(Received 19 September 2007; accepted 8 November 2007; published online 9 January 2008)

\begin{abstract}
The ultrafast dynamics of HDO: $\mathrm{D}_{2} \mathrm{O}$ ice $\mathrm{Ih}$ at $180 \mathrm{~K}$ is studied by midinfrared ultrafast pump-probe spectroscopy. The vibrational relaxation of $\mathrm{HDO}: \mathrm{D}_{2} \mathrm{O}$ ice is observed to proceed via an intermediate state, which has a blueshifted absorption spectrum. Polarization resolved measurements reveal that the intermediate state is part of the intramolecular relaxation pathway of the HDO molecule. In addition, slow dynamics on a time scale of the order of $10-100 \mathrm{ps}$ is observed, related to thermally induced collective reorganizations of the ice lattice. The transient absorption line shape is analyzed within a Lippincott-Schroeder model for the $\mathrm{OH}$-stretch potential. This analysis identifies the main mechanism behind the strong spectral broadening of the $v_{\mathrm{OH}}=1$ $\rightarrow 2$ transition. (C) 2008 American Institute of Physics. [DOI: 10.1063/1.2820765]
\end{abstract}

\section{INTRODUCTION}

While the ultrafast dynamics of liquid water has been studied in great detail, time-resolved studies on the properties of ice have so far been limited. In the study of water, vibrational spectroscopies have proven to be very useful, in particular, because of the sensitivity of vibrations to local hydrogen bonding. The phase transition associated with freezing HDO: $\mathrm{D}_{2} \mathrm{O}$ water leads to a strong narrowing of the $\mathrm{OH}$-stretch absorption, from a width of $200 \mathrm{~cm}^{-1}$ in water at $298 \mathrm{~K}$ to only $50 \mathrm{~cm}^{-1}$ in ice at $180 \mathrm{~K}$. This narrowing results from the transition from a disordered liquid phase to an ordered crystalline phase. The water molecules in ice are positioned on a regular hexagonal lattice, hydrogen bonded to four nearest neighbors. This ordering leads to a strong reduction of the spread in hydrogen bond lengths. Since there exists a strong correlation between the $\mathrm{OH}$-stretch absorption frequency and the hydrogen bond length, the narrowing of the hydrogen bond distribution also leads to a narrowing of the $\mathrm{OH}$ vibrational spectrum.

Despite the relative uniformity in the $\mathrm{OH} \cdots \mathrm{O}$ hydrogen bonds, the structure and hydrogen bonding properties of ice Ih are only seemingly simple topics, and have been puzzling physicists and chemists for a long time. Although a proton or deuteron is found along every $\mathrm{O} \cdots \mathrm{O}$ line in an ice crystal, the individual hydrogen bonds and covalent bonds are positioned randomly under constraints known as "ice rules." The ice lattice is therefore said to be proton disordered. Combined theoretical and experimental scattering studies on ice $I h$ show that, as a result of proton disorder, individual water molecules deviate from their lattice sites by static displacements of the oxygen positions. ${ }^{2-4}$ From a spectroscopic point of view, it was suggested that proton disorder should lead to inhomogeneous broadening of the linear $\mathrm{OH}$-stretch spectrum in $\mathrm{HDO}: \mathrm{D}_{2} \mathrm{O} .{ }^{5}$

The inhomogeneous broadening of the $\mathrm{OH}$-stretch absorption in HDO: $\mathrm{D}_{2} \mathrm{O}$ ice was confirmed by the first ultrafast spectroscopic study on ice, using picosecond IR

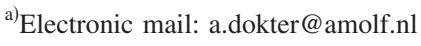

pump-probe spectroscopy. ${ }^{6}$ This work also showed that the $v_{\mathrm{OH}}=1 \rightarrow 2$ transition of ice is strongly broadened compared the narrow $v_{\mathrm{OH}}=0 \rightarrow 1$ transition. While the $v_{\mathrm{OH}}=0 \rightarrow 1$ transition has a full width at half maximum (FWHM) of only $50 \mathrm{~cm}^{-1}$ at $180 \mathrm{~K}$, the $v_{\mathrm{OH}}=1 \rightarrow 2$ transition has a width of $350 \mathrm{~cm}^{-1}$. Theoretical modeling of the (linear) $v_{\mathrm{OH}}=0 \rightarrow 1$ absorption spectrum of ice still is an ongoing field of research. $^{7,8}$ To understand the nonlinear pump-probe spectrum, we need additional understanding of the $v_{\mathrm{OH}}=1 \rightarrow 2$ absorption spectrum.

The line shape of the $v_{\mathrm{OH}}=1 \rightarrow 2$ transition is of importance, since it may contain information on the $\mathrm{OH}$-stretch potential of a hydrogen bonded water molecule. This potential is usually described as an asymmetric double well potential with a barrier height being dependent on the precise length of the hydrogen bond $R,{ }^{5,9-11}$ as illustrated in Fig. 1 . The high energy minimum in the potential refers to the state in which a water molecule has undergone autodissociation forming a hydronium-hydroxide ion pair. The precise barrier height between the two states is strongly dependent on the hydrogen bond coordinate $R$. As will be discussed, the

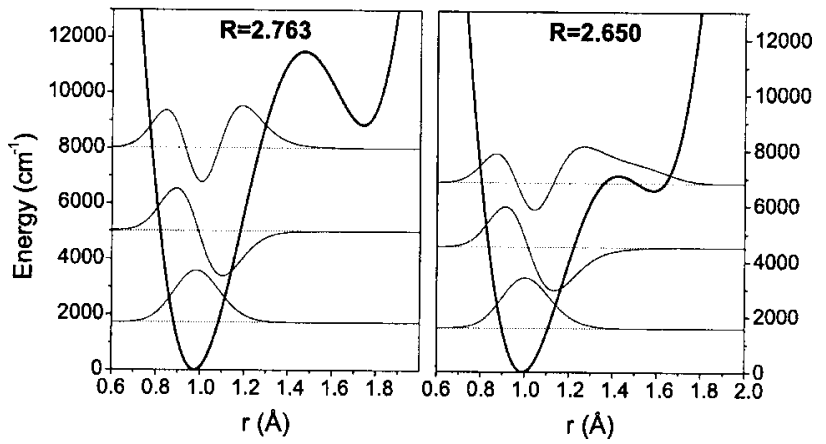

FIG. 1. Lippincott-Schroeder potential and wavefunctions for the $\mathrm{OH}$ stretch coordinate $r$. The potential has the form of an asymmetric double well, for which the high energy minimum refers to a proton transferred state by formation of a hydronium-hydroxide ion pair. The left panel shows the potential for the equilibrium $\mathrm{O} \cdots \mathrm{O}$ hydrogen bond length of an ice crystal at $180 \mathrm{~K}(R=2.763 \AA)$. The right panel shows the potential for a shortened hydrogen bond $(R=2.650 \AA)$, for which the wavefunction is delocalized over the two wells. 
$v_{\mathrm{OH}}=1 \rightarrow 2$ transition can strongly broaden as soon as the $v_{\mathrm{OH}}=2$ state becomes close in energy to the dissociation barrier.

In this article we report on spectrally resolved subpicosecond midinfrared experiments on $\mathrm{HDO}: \mathrm{D}_{2} \mathrm{O}$ ice $I h$. The experiments characterize the vibrational relaxation pathway and the transient absorption line shape. The transient line shape is analyzed within a Lippincott-Schroeder model (LS model), which allows us to identify the main broadening mechanism for the $v_{\mathrm{OH}}=1 \rightarrow 2$ transition.

\section{EXPERIMENT}

We performed ultrafast midinfrared pump-probe spectroscopy on the $\mathrm{O}-\mathrm{H}$ stretch vibration of diluted HDO in $\mathrm{D}_{2} \mathrm{O}$ at $180 \mathrm{~K}$. Isotopically diluted water samples $(1: 40$ $\mathrm{H}_{2} \mathrm{O}: \mathrm{D}_{2} \mathrm{O}$ ) are used to prevent the signals to be affected by intermolecular resonant energy transfer of the $\mathrm{OH}$-stretch vibrations, and to limit heating of the sample. Water was inserted between two $\mathrm{CaF}_{2}$ windows without spacer and inserted into a closed cycle cryostat (CTI-Cryogenics). The sample was cooled to $180 \mathrm{~K}$. The linear absorption is found to be isotropic for all polarization angles, which shows that the sample is polycrystalline with crystalline domains much smaller than the laser focus. No local order or dipole alignment is present in the sample.

In the experiment, a first intense midinfrared light pulse excites the $\mathrm{OH}$ oscillators, inducing transmission changes of a weak second time-delayed probe pulse. After excitation, the transmission is increased at frequencies matching the $v_{\mathrm{OH}}=0 \rightarrow 1$ transition (because of ground state depletion of the $\mathrm{OH}$-stretch vibration and stimulated emission out of the $v_{\mathrm{OH}}=1$ state), while the transmission is decreased at the more redshifted frequencies matching the $v_{\mathrm{OH}}=1 \rightarrow 2$ transition (because of absorption by the induced population in the $v_{\mathrm{OH}}=1$ state).

The femtosecond midinfrared light source has been described previously elsewhere. ${ }^{12}$ The generated probe pulses are spectrally broad compared to the pump pulses $\left(150 \mathrm{~cm}^{-1}\right.$ and $80 \mathrm{~cm}^{-1}$ FWHM, respectively), and the time resolution is $\sim 150 \mathrm{fs}$. After reflecting the probe beam off a glass window to lower its intensity by $95 \%$, the pump and probe beams are overlapped and focussed at the sample inside the cryostat. The transmitted probe beam is sent through a polarizer and spectrally resolved on a nitrogen-cooled $\mathrm{HgCdTe}$ detector array using a polychromator. Using an automated rotatable mount, the polarizer can be set either parallel or perpendicular with respect to the pump polarization, and at each delay point both probe components are detected consecutively. In this way, pump induced transmission changes for both the probe component polarized parallel $\left(\Delta \alpha_{\|}\right)$and perpendicular $\left(\Delta \alpha_{\perp}\right)$ with respect to the pump polarization are measured.

The two polarization components are used to construct the so-called rotation-free signal $\Delta \alpha_{\mathrm{RF}}(\omega, t)$ and the anisotropy parameter $R(\omega, t)$ (depending both on the probe frequency $\omega$ and the probe time delay $t)$. The rotation-free signal is given by

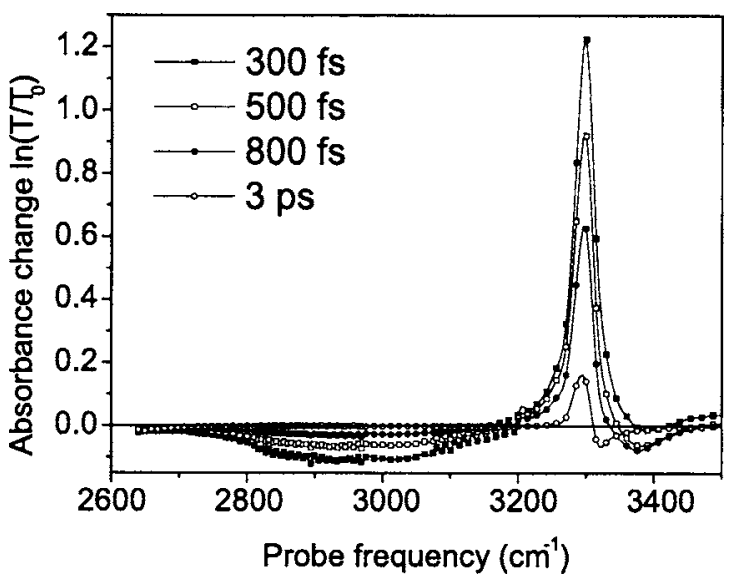

FIG. 2. Pump induced absorbance change plotted as a function of probe frequency at probe delay $t$ of $0.3,0.5,0.8$, and $3 \mathrm{ps}$. The negative induced absorption signal is strongly broadened compared to the positive bleaching signal. The absorbance change is defined as $\ln \left(T / T_{0}\right)$, where $T$ is the transmittance after a delay $t$ of the excitation by the pump, and $T_{0}$ the transmittance in the absence of pump excitation.

$$
\Delta \alpha_{\mathrm{RF}}(\omega, t)=\frac{1}{3}\left(\Delta \alpha_{\|}+2 \Delta \alpha_{\perp}\right) .
$$

This signal is not affected by molecular reorientation. The anisotropy parameter is defined as

$$
R(\omega, t)=\frac{\Delta \alpha_{\|}-\Delta \alpha_{\perp}}{\Delta \alpha_{\|}+2 \Delta \alpha_{\perp}} .
$$

This parameter reflects only anisotropic absorption changes. Its amplitude will not decay as long as molecules keep the orientational information of the pump process, by which preferentially molecules parallel to the pump polarization are excited. For an ensemble of randomly oriented dipole oscillators, the maximum anisotropy value equals 0.4 .

\section{RELAXATION DYNAMICS OF VIBRATIONALLY EXCITED ICE}

Figure 2 shows the transient absorption of HDO ice $\left(\mathrm{H}_{2} \mathrm{O}: \mathrm{D}_{2} \mathrm{O}=1: 40\right)$ at several delays after vibrational excitation by the pump pulse. The transient line shape consists mainly of a narrow bleaching peak at the $\mathrm{OH}$-stretch absorption around $3300 \mathrm{~cm}^{-1}$, and a very broad induced absorption around $3000 \mathrm{~cm}^{-1}$. This large difference in linewidth was previously observed by picosecond IR hole-burning spectroscopy, ${ }^{6}$ the induced absorption peak being over a factor of 5 broader than the bleaching contribution.

We determine the $\mathrm{OH}$-stretch vibrational relaxation rate by measuring the decay of the induced absorption in time. The spectral region of the induced absorption $\left(2800-3200 \mathrm{~cm}^{-1}\right)$ contains no other absorbing species, making the signal solely proportional to the population of the $v_{\mathrm{OH}}=1$ vibrational state. In agreement with previous work, ${ }^{13}$ we find that at all frequencies the induced absorption decays monoexponentially with a time constant of $420 \pm 20 \mathrm{fs}$.

Interestingly, the induced bleaching around $3300 \mathrm{~cm}^{-1}$ decays differently than the induced absorption around $3000 \mathrm{~cm}^{-1}$. This is illustrated in Fig. 3, in which the transient absorbance change at the peak of the bleaching is compared with the decay of the induced absorption. Both curves are 


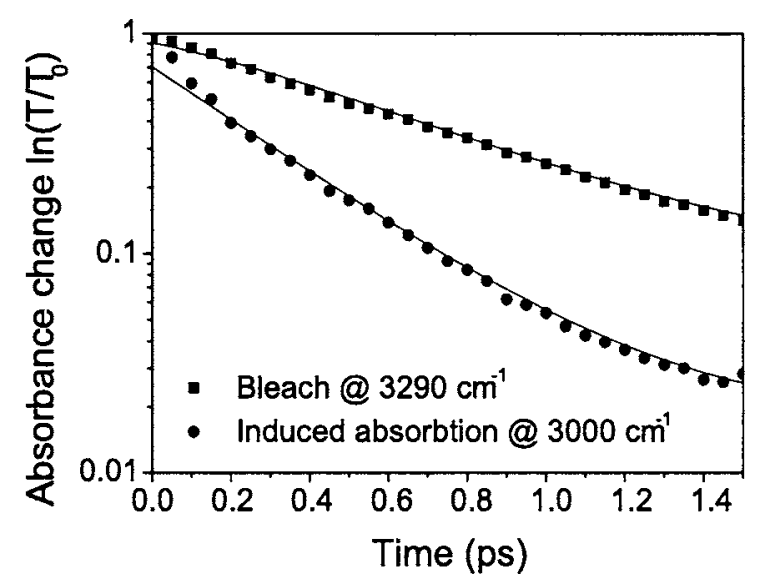

FIG. 3. Pump induced absorbance change plotted as a function of probe delay at $3290 \mathrm{~cm}^{-1}$ (center frequency bleaching) and $3000 \mathrm{~cm}^{-1}$ (center frequency induced absorption). Both curves are scaled to equal amplitude and sign to facilitate comparison of the dynamics. The relaxation is faster for the induced absorption than for the bleaching.

scaled to equal amplitude and sign to facilitate easy comparison of the dynamics. The figure clearly shows that the sharp bleaching feature around $3300 \mathrm{~cm}^{-1}$ decays at about half the rate of the induced absorption.

A more careful examination of the transients shown in Fig. 2 shows additional spectral dynamics, namely, the appearance at about $400 \mathrm{fs}$ of an induced absorption around $3380 \mathrm{~cm}^{-1}$. At later delays, the transient spectrum takes the form of a thermal difference spectrum, which also results in induced absorption, however, at $3320 \mathrm{~cm}^{-1}$. This thermal signal $\Delta \alpha_{T}(\omega, t)$ arises from effects of heating of the sample due to the intense pump pulse, i.e., the effect on the $\mathrm{OH}-$ stretch absorption of population of low-frequency modes after vibrational relaxation. ${ }^{14}$

We find that the induced absorption at $3380 \mathrm{~cm}^{-1}$ grows in at a rate that equals the vibrational relaxation, and disappears when the relatively slow decay of the bleaching at $3300 \mathrm{~cm}^{-1}$ is also complete. These corresponding time scales suggest that both the absorption at $3380 \mathrm{~cm}^{-1}$ and the longer lifetime of the bleaching at $3300 \mathrm{~cm}^{-1}$ arise from the same effect, namely, that the vibrational relaxation proceeds via an intermediate state.

To determine the spectral signature of this intermediate state, we fit the data to a simple kinetic model, as illustrated in Fig. 4. State 0 comprises the thermal ground state of the water molecules, and state 1 represents the thermal ground state 0 plus one quantum in the $\mathrm{OH}$-stretch coordinate. Vibrational relaxation from the initially populated state 1 proceeds via a nonthermal intermediate level $0^{*}$ with a different associated cross section. This model does not make any assumptions on the nature or energy of the intermediate state. The total rotation-free pump-probe signal $\Delta \alpha(\omega, t)$ can be written in terms of the populations $N_{1}, N_{0}^{*}$ of the levels in Fig. 4 of the initially excited molecules only, the absorption cross-sections $\sigma$ between these levels, and the total concentration of absorbing molecules C. ${ }^{14}$

$$
\Delta \alpha(\omega, t) \propto \Delta \sigma_{P P}(\omega) N_{1}(t)+\Delta \sigma_{01} *(\omega) N_{0}^{*}(t)+\Delta \alpha_{T}(\omega, t),
$$

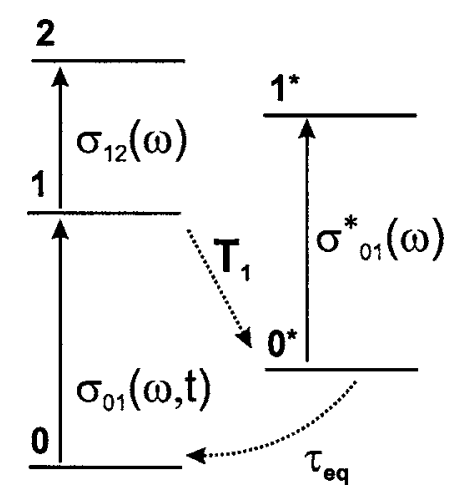

FIG. 4. Schematic overview of the model that describes the population dynamics of excited HDO molecules. Relaxation from the excited $v_{\mathrm{OH}}=1$ state proceeds via an intermediate level $0^{*}$ with associated $\mathrm{OH}$-stretch cross section $\sigma_{01}^{*}(\omega)$ that is different from the cross section $\sigma_{01}^{*}(\omega, t)$ in the ground state.

$$
\begin{aligned}
& \Delta \sigma_{\mathrm{PP}}(\omega)=\left[\sigma_{12}(\omega)-2 \sigma_{01}(\omega, 0)\right], \\
& \Delta \sigma_{01} *(\omega)=\left[\sigma_{01}^{*}(\omega)-\sigma_{01}(\omega, 0)\right], \\
& \Delta \alpha_{T}(\omega, t)=C\left[\sigma_{01}(\omega, t)-\sigma_{01}(\omega, 0)\right] .
\end{aligned}
$$

The time dependencies of the populations and $\Delta \alpha_{T}$ are described in Appendix A. Fitting our data to the kinetic model gives the spectra $\Delta \sigma_{\mathrm{PP}}(\omega)$ and $\Delta \sigma_{01} *(\omega)$ and the lifetime of the intermediate level $\tau_{\mathrm{eq}}$, which is found to be $390 \pm 40 \mathrm{fs}$. Figure 5 shows the fitted curves overlayed with the measured data for five frequencies up to $3 \mathrm{ps}$.

In Fig. 6 the spectra $\Delta \sigma_{\mathrm{PP}}(\omega)$ and $\Delta \sigma_{01} *(\omega)$ are shown. $\Delta \sigma_{\mathrm{PP}}(\omega)$ equals the pure pump-probe line shape at time $t$ $=0$, before any vibrational relaxation has occurred. We find a FWHM of the bleaching line of $44 \mathrm{~cm}^{-1}$, and $300 \mathrm{~cm}^{-1}$ for the induced absorption. $\Delta \sigma_{01}{ }^{*}(\omega)$ is the pump-probe spectrum associated with the intermediate level.

An important question regarding the nature of the intermediate level is whether the intermediate state absorption is solely due to molecules that were excited, or that also nonexcited molecules contribute to the intermediate state absorption. In the first case vibrational relaxation leads to popula-

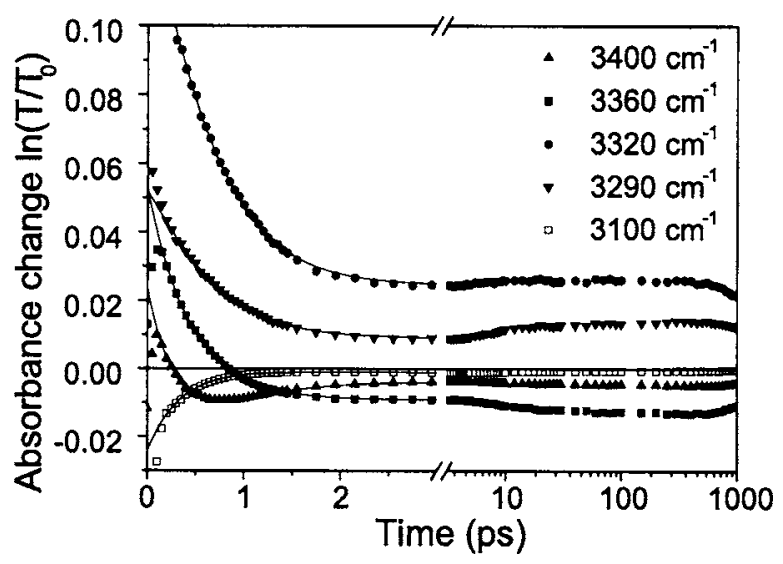

FIG. 5. Pump induced absorbance change plotted as a function of probe delay at various probe frequencies. From 3 ps onwards absorbance changes are observed related to slow collective rearrangements of the hydrogen bonded crystal lattice. 


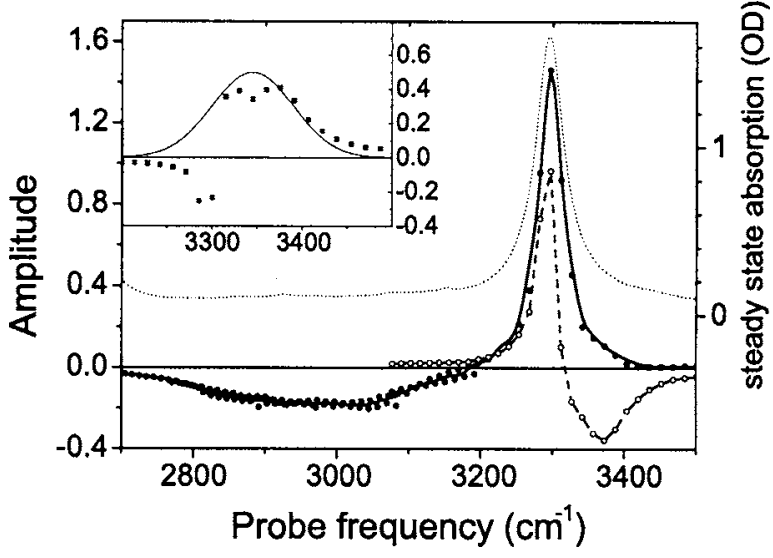

FIG. 6. Pure pump-probe spectrum $\Delta \sigma_{\mathrm{PP}}(\omega)(\boldsymbol{Q}$, solid line) and intermediate state pump-probe spectrum $\Delta \sigma_{0} *(\omega)$ ( $\bigcirc$ dashed line). For comparison, the linear $\mathrm{OH}$-stretch absorption spectrum of isotopically diluted (1:40) ice at $180 \mathrm{~K}$ is shown (dotted line). Since our probe spectrum is not broad enough to cover the full spectral range of interest, the displayed data result from merging three spectra measured separately with the probe wavelength centered at 2800, 3000, and $3300 \mathrm{~cm}^{-1}$. The inset shows the extracted linear absorption of the intermediate state $\sigma_{0}$ *

tion of modes localized near the excited $\mathrm{OH}$ group that change its $\mathrm{OH}$-stretch absorption frequency, while in the latter case vibrational relaxation populates delocalized modes that affect the $\mathrm{OH}$-stretch absorption frequencies of several molecules, including molecules that were not excited.

We can distinguish between these two cases by examining the anisotropic signal as shown in Fig. 7. In the spectral region of the broad induced absorption, the intermediate state has no cross section, and we measure only the anisotropy value of the molecules of which the $\mathrm{OH}$-stretch vibration is excited. Within the accuracy of our experiment, this anisotropy is shown to retain a constant value of 0.3 . This value is somewhat lower than the expected value of 0.4 , probably because of scattering of the probe light. The constant value shows that during vibrational excitation the $\mathrm{OH}$-stretch transition dipole in the first excited state does not change orientation, as expected for a rigid crystalline structure as ice. In spectral regions where also the intermediate state has signifi-

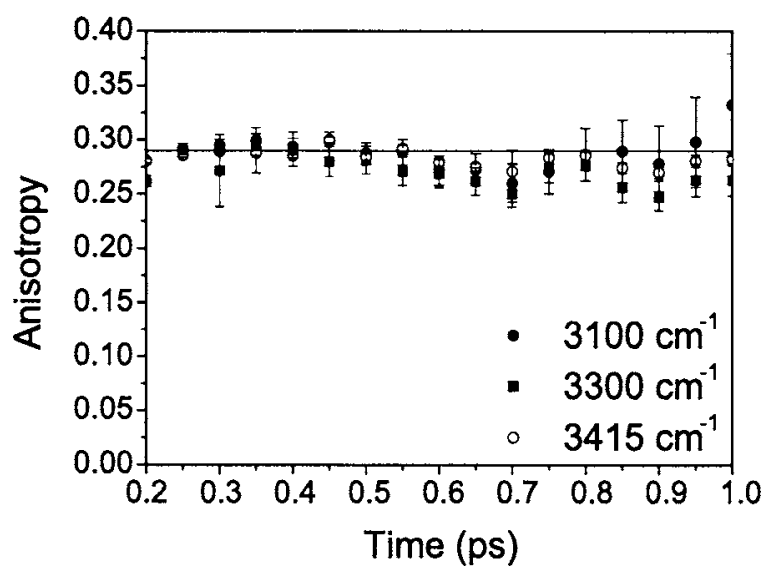

FIG. 7. Anisotropy decay at various probe frequencies. We observe no significant decay of the anisotropy within the first $1 \mathrm{ps}$. At the probe frequency of $3415 \mathrm{~cm}^{-1}(\mathrm{O})$ only the intermediate state absorbs. The intermediate state anisotropy equals that of the bleaching $(\boldsymbol{\square})$ and induced absorption (O) contributions. cant cross section, we measure the exact same anisotropy, also when most population has been transferred to the intermediate level from 500 fs onwards. The transition dipole of the intermediate state absorption therefore has to be parallel to the transition dipole of the initial state, otherwise the anisotropy of the intermediate state would have been lower. This demonstrates that the intermediate state absorption originates from spectral changes of the excited molecules only, following their vibrational relaxation, and is likely related to intramolecular relaxation.

To obtain the linear absorption spectrum of molecules in the intermediate state $\sigma_{01}^{*}(\omega)$, we need to add the linear absorption spectrum of molecules in the ground state $\sigma_{01}(\omega)$ to the intermediate state pump-probe spectrum $\Delta \sigma_{01} *(\omega)$ with the correct proportionality constant [see Eq. (3)]. If we assume that $\sigma_{12}(\omega)$ has little absorption above $3200 \mathrm{~cm}^{-1}$ we can write $\sigma_{01}^{*}(\omega)=\Delta \sigma_{01} *(\omega)-\frac{1}{2} \Delta \sigma_{\mathrm{PP}}(\omega)$ for $\omega>3200 \mathrm{~cm}^{-1}$. The intermediate state spectrum obtained in this way is shown in the inset of Fig. 6.

Compared to the initial state, the intermediate state absorption is broader with a FWHM of $100 \pm 20 \mathrm{~cm}^{-1}$ and blueshifted to $3350 \pm 20 \mathrm{~cm}^{-1}$. This blueshift can result both from intramolecular relaxation to the bending mode and from relaxation to local hydrogen bond modes. Excitation of the bending vibration dynamically decouples the $\mathrm{OH}$-stretch motion from the hydrogen bond, resulting in hydrogen bond weakening and a blueshift of the $\mathrm{OH}$-stretch frequency. $\mathrm{Re}$ cently, a blueshifted intermediate state associated with the excitation of a bending quantum was also reported for liquid water. ${ }^{15}$ The $\mathrm{OH}$-stretch absorption spectrum is known to blueshift with increasing temperature, which is predominantly caused by the weakening of the hydrogen bonds. A local temperature rise by about $200 \mathrm{~K}$ can be achieved by the energy of one relaxed vibrational quantum (calculated from molecular density and specific heat, in the limit where the energy is shared by one HDO molecule and its four next neighbors only). ${ }^{6}$

As a final point of interest, the thermal pump-probe spectrum $\Delta \alpha_{T}(\omega, t)$ shows some remarkable dynamics over longer timescales, as displayed in Fig. 5. The dynamics has multiexponential character, as is often observed for processes involving heat diffusion. The strongest increase in the thermal signal is observed from 3 to 40 ps (exponential time constant of $5 \pm 1 \mathrm{ps}$ ). Further, small signal increases are observed up to $100-400 \mathrm{ps}$, after which the thermal signal starts to decay as a result of cooling.

In the context of superheating experiments of ice reported in literature, it has been suggested that these slow dynamics are related to partial melting of the ice lattice. ${ }^{16}$ In our experiment a melting process is, however, not likely to occur. The amount of heat dumped in the sample is very limited in view of the low concentration of $\mathrm{OH}$-stretch absorbers, causing the sample to be heated by a few degrees kelvin only after completion of the vibrational relaxation of the intermediate state. The longer time scale dynamics are likely caused by collective reorganization of the ice lattice (e.g., thermal expansion). Since these dynamics involve very low-frequency modes in the hydrogen bond coordinates, the thermal equilibrium positions for these coordinates are 
reached fairly slowly. Cooling of the focal region to the surrounding temperature will cause the thermal signal to decay entirely eventually, as seen from 400 ps onwards.

\section{ABSORPTION LINE SHAPE OF VIBRATIONALLY EXCITED ICE}

\section{A. OH-stretch frequency dependence of the hydrogen bond}

A striking feature of the pure pump-probe spectrum $\Delta \sigma_{\mathrm{PP}}(\omega)$ of ice is the huge difference in linewidth between the $v_{\mathrm{OH}}=1 \rightarrow 2$ and $v_{\mathrm{OH}}=0 \rightarrow 1$ transitions. A possible explanation for the huge width of the $v_{\mathrm{OH}}=1 \rightarrow 2$ can be found from the effect of the hydrogen bond on the $\mathrm{OH}$-stretch vibrational potential. This $\mathrm{OH}$-stretch vibrational potential is usually described as an asymmetric double well potential with a barrier height being dependent on the precise length of the hydrogen bond $R ., 5,10$

We describe the relation between $\mathrm{OH}$-stretch frequency and hydrogen bond length by a Lippincott-Schröder model, ${ }^{9}$ which has been applied successfully in the description of the transient spectrum of liquid water. ${ }^{11,17}$ The LS potential for the $\mathrm{O}-\mathrm{H}$ coordinate of the water molecule is given by

$$
\begin{aligned}
V_{\mathrm{LS}}(r, R)= & D_{a}\left[1-e^{-n_{a}\left(r-r_{0}\right)^{2} / 2 r}\right] \\
& +D_{b}\left[1-e^{-n_{b}\left(R-r-r_{0}\right)^{2} / 2(R-r)}\right]+V_{0}(r, R),
\end{aligned}
$$

where $V_{0}(r, R)$ is a potential term that sets the absolute minimum of the full potential at 0 for all $(r, R)$. We adopt the same parameters of $V_{\mathrm{LS}}(r, R)$ as used previously in the description of the transient spectrum of liquid water, ${ }^{17}$ except for the parameter $n_{a}\left(10.0 \AA^{-1}\right)$, which was slightly upscaled to yield the correct central $\mathrm{OH}$-stretch absorption frequency at $180 \mathrm{~K}\left(3290 \mathrm{~cm}^{-1}\right)$ at the experimentally determined equilibrium hydrogen bond length of $2.76 \AA \AA^{4}$. The energy $D_{a}$ equals the $\mathrm{O}-\mathrm{H}$ binding energy of water of $38750 \mathrm{~cm}^{-1}$ $(4.8 \mathrm{eV}) . r_{0}$ represents the $\mathrm{O}-\mathrm{H}$ bond length in the gas phase in the absence of a hydrogen bond $(0.97 \AA)$. Parameters $n_{b}$ $\left(16.5 \AA^{-1}\right)$ and $D_{b}\left(25000 \mathrm{~cm}^{-1}\right)$ are determined by the relations between the $\mathrm{O}-\mathrm{H}$ bond length $R$, the $\mathrm{O}-\mathrm{H}$ stretch vibrational frequency, and the oxygen-oxygen distance $R .^{9,17}$

The LS potential is illustrated in Fig. 1. The low energy minimum refers to the normal hydrogen bonded configuration of the water molecule. The high energy minimum refers to the configuration in which the hydrogen bond donating water molecule has undergone autodissociation, forming a hydroxide and hydronium ion pair by a proton transfer event to the neighboring water molecule. For strong (short) hydrogen bonds, the energy barrier between these two configurations gets substantially lowered. When the barrier height is of the order of the energy of the $v_{\mathrm{OH}}=2$ state of the $\mathrm{OH}$ stretching vibration, the vibrational wavefunction gets delocalized over the two wells, reflecting the possibility of proton transfer. The precise energy of the vibrational state will be highly sensitive to the precise height of the energy barrier and, thus, to the hydrogen bond coordinate. In other words, small variations in $R$ can give a large spread in $v_{\mathrm{OH}}=2$ vibrational energies, and therefore lead to a significant broadening of the $v_{\mathrm{OH}}=1 \rightarrow 2$ absorption. This mechanism has been shown to adequately explain the line shape of vibrationally excited liquid water. ${ }^{11,17}$ We will test whether a similar mechanism can explain the huge broadening of the $v_{\mathrm{OH}}=1 \rightarrow 2$ transition in ice.

We will estimate the distribution in hydrogen bond lengths of an ice $I h$ crystal at $180 \mathrm{~K}$, and how these hydrogen bonds will be partly modulated in time by the presence of low-frequency phonons (Sec. IV B). With these ingredients we can calculate the dipole time correlation functions (Sec. IV C) that determine the ground state and excited state absorptions. Finally, we discuss the transient absorption line shapes resulting from these calculations (Sec. IV D).

\section{B. Hydrogen bond distribution and dynamics}

The linewidth of the $v_{\mathrm{OH}}=0 \rightarrow 1$ transition equals $50 \mathrm{~cm}^{-1}$ at $180 \mathrm{~K}$. This linewidth cannot be explained from the lifetime of the $v_{\mathrm{OH}}=1$ state $\left(T_{1}^{v=1}=0.42 \mathrm{ps}\right)$. Lifetime broadening leads to a Lorentzian absorption profile for each oscillator, with the FWHM linewidth being only $13 \mathrm{~cm}^{-1}$ $\left[1 /\left(2 \pi T_{1}^{v=1}\right)\right]$. The $\mathrm{OH}$-stretch linewidth must therefore be additionally broadened by the dynamics and the spread in length of the hydrogen bonds that occur in the ice crystal. To calculate the transient line shape, we need to know these hydrogen bond dynamics and length distribution. We can then relate these to $\mathrm{OH}$-stretch frequencies and cross sections by the LS model as shown in the previous paragraph.

Proton disorder in the ice crystal leads to a static spread in oxygen positions in the ice lattice, even at low temperatures. Therefore, a static spread $\Delta_{R \text {,stat }}$ in the $\mathrm{O} \cdots \mathrm{O}$ hydrogen bond lengths $R$ exists in the ice lattice, which is unfortunately very hard to deduce from experimental scattering studies. ${ }^{4}$ This static distribution in hydrogen bond lengths will determine the inhomogeneous broadening of the ice $\mathrm{OH}$-stretch absorption. Picosecond IR hole-burning experiments reported some inhomogeneous broadening of the $\mathrm{OH}-$ stretch band, however, only with a very narrow distribution (spectral holes were separated by at most $15 \mathrm{~cm}^{-1}$, corresponding to a standard deviation in hydrogen bond lengths of $\sim 0.4 \mathrm{pm}$ in our LS model, ${ }^{6}$ which is small compared to the standard deviation of $1.5 \mathrm{pm}$ of room temperature liquid water ${ }^{17}$ ). A recent theoretical study ${ }^{4}$ also shows a very small standard deviation of $0.7 \mathrm{pm}$. We therefore assume in our calculation a Gaussian hydrogen bond distribution $f_{\text {stat }}(R)$ of fixed standard deviation $\Delta_{R \text {,stat }}=0.4 \mathrm{pm}$ around equilibrium bond length $R_{0}=2.76 \AA$, in close agreement with the small hydrogen bond spreads found both in theory and experiment. Within the LS model, this hydrogen bond distribution leads to a FWHM OH-stretch $v_{\mathrm{OH}}=0 \rightarrow 1$ linewidth of only $20 \mathrm{~cm}^{-1}$. This is much narrower than the experimentally measured linewidth of $50 \mathrm{~cm}^{-1}$ at $180 \mathrm{~K}$, for which proton disorder alone can therefore not account.

Another line broadening mechanism results from lattice dynamics that modulate the $\mathrm{O} \cdots \mathrm{O}$ hydrogen bond, ${ }^{18}$ and thereby the $\mathrm{OH}$-stretch frequency. Thermally excited phonons will modulate the hydrogen bond coordinate by a certain amplitude and at a characteristic time scale. In our model we will assume that the presence of phonons will lead to a diffusional modulation of the hydrogen bond coordinate 
(see Appendix B). We thereby do not model certain phonon satellite band progressions that are observed in the $\mathrm{OH}$ stretch spectrum of isotopically dilute ice. ${ }^{19}$ These are associated with combined transitions to higher energy underdamped nonthermal phonons.

We need to make assumptions about the amplitude and rate of the hydrogen bond fluctuations. The amplitudes of the fluctuations are related to phonon amplitudes, which are typically smaller than $2 \mathrm{pm} .{ }^{20}$ This sets an upper limit to the width $\Delta_{R \text {,dyn }}$ of the hydrogen bond distribution that is dynamically sampled by the hydrogen bond length fluctuations. The rate of the fluctuations is set by a diffusional constant for the hydrogen bond length modulation process. This diffusional constant $D$ is directly related to the decay constant $\tau_{c}$ of the frequency correlations [see Eq. (B11)]. We assume that $\tau_{c} \gtrsim h /\left(k_{B} T\right)=0.27 \mathrm{ps}$, i.e., the frequency correlations cannot decay faster than the oscillation period of the average occupied thermal mode. We choose $\Delta_{R \text {,dyn }}=1 \mathrm{pm}$, and adjust the diffusion constant $D$ by a fit of the calculated line shape to the experimental spectrum (see Sec. IV D). This results in $D=30 \mathrm{pm}^{2} / \mathrm{ps}$ and $\tau_{c}=0.3 \mathrm{ps}$.

\section{Line shape calculation}

Following the Wiener-Khintchine theorem, the absorption spectrum for a transition from state $i \rightarrow j$ is the Fourier transform of the dipole time correlation function.

$$
S_{i j}(\omega)=\frac{1}{2 \pi} \int_{-\infty}^{\infty} d t \cdot e^{-i \omega t}\left\langle\tilde{\mu}_{i j}(0) \tilde{\mu}_{i j}(t)\right\rangle .
$$

$\tilde{\mu}_{i j}(t)$ is the molecular dipole operator, and \langle\rangle denotes ensemble averaging over all oscillators. ${ }^{21}$ Modeling the line shape thus involves the calculation of the dipole time correlation function $C_{i j}(t)=\left\langle\tilde{\mu}_{i j}(0) \tilde{\mu}_{i j}(t)\right\rangle$. This correlation function is determined by time evolution of the dipole moments, which is determined by the transition frequencies, transition dipole moments, and the vibrational lifetimes of the involved states. The transition frequencies and transition dipole moments depend on the hydrogen bond coordinate $R$, as calculated in Appendix B. The spread and dynamics of the hydrogen bond coordinate $R$ was discussed in the previous section.

We assume that the pump and probe pulses are short compared to the system dynamics [impulsive pump-probe (IPP) limit], and take the excited state and ground state dynamics fully uncorrelated and in thermal equilibrium. We treat the vibrational state spectrum of water as an effective three-level system. In that case only $\widetilde{\mu}_{01}$ and $\tilde{\mu}_{12}$ have significant amplitude. The pump-probe signal at zero probe delay $S_{\text {IPP }}(\omega, 0)$ can then be related to the linear response of Eq. (6) by

$$
S_{\text {IPP }}(\omega, 0) \propto 2 S_{01}(\omega)-S_{12}(\omega),
$$

referring to the bleaching and induced absorption contributions, respectively. The line shape calculation is discussed in detail in Appendix B.

\section{Discussion of the calculated transient line shapes}

In Fig. 8 the calculated line shapes for the $v_{\mathrm{OH}}=1 \rightarrow 2$ and the $v_{\mathrm{OH}}=0 \rightarrow 1$ transition (solid lines) are shown when

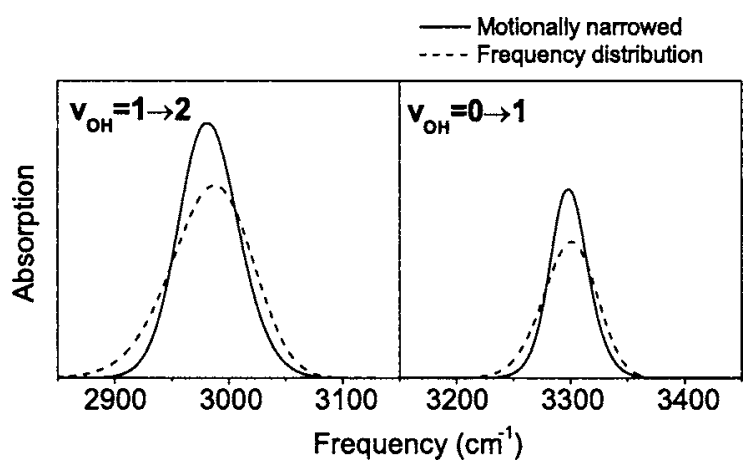

FIG. 8. Absorption line shapes for the $v_{\mathrm{OH}}=1 \rightarrow 2$ and the $v_{\mathrm{OH}}=0 \rightarrow 1$ transitions (solid lines) calculated when assuming a frequency distribution (dotted lines) for a Gaussian hydrogen bond length distribution of standard deviation $\Delta_{R, \text { dyn }}=1 \mathrm{pm}$ (i.e., excluding inhomogeneous and lifetime broadenings).

assuming a frequency distribution for a Gaussian hydrogen bond length distribution of standard deviation $\Delta_{R, \text { dyn }}=1 \mathrm{pm}$ and a mean of $R_{0}=2.76 \AA$ (dotted lines). The hydrogen bonds diffuse with a diffusional constant $D=30 \mathrm{pm}^{2} / \mathrm{ps}$ (see Appendix B), and lifetime broadening is not included by taking the vibrational lifetimes infinite.

The frequency modulations due to phonons clearly result in motional narrowing of the spectral lines. The degree of motional narrowing is similar for the $v_{\mathrm{OH}}=0 \rightarrow 1$ and $v_{\mathrm{OH}}$ $=1 \rightarrow 2$ absorptions.

By including inhomogeneous broadening and lifetime broadening in the calculation, we can calculate the full transient absorption lineshape according to Eq. (7). The only remaining adjustable parameter is the vibrational lifetime $T_{1}^{v=2}$ of the $v_{\mathrm{OH}}=2$ state. In Fig. 9 (dotted line) the line shape is calculated when $T_{1}^{v=2}=T_{1}^{v=1} / 2$ (as would be expected for a harmonic oscillator, based on the larger dipole coupling matrix element between the $v_{\mathrm{OH}}=1,2$ states compared to the $v_{\mathrm{OH}}=0,1$ states). While the calculated line shape is reasonable for the $v_{\mathrm{OH}}=0 \rightarrow 1$ transition, it gives a poor description of the experimentally measured $v_{\mathrm{OH}}=1 \rightarrow 2$ absorption line shape.

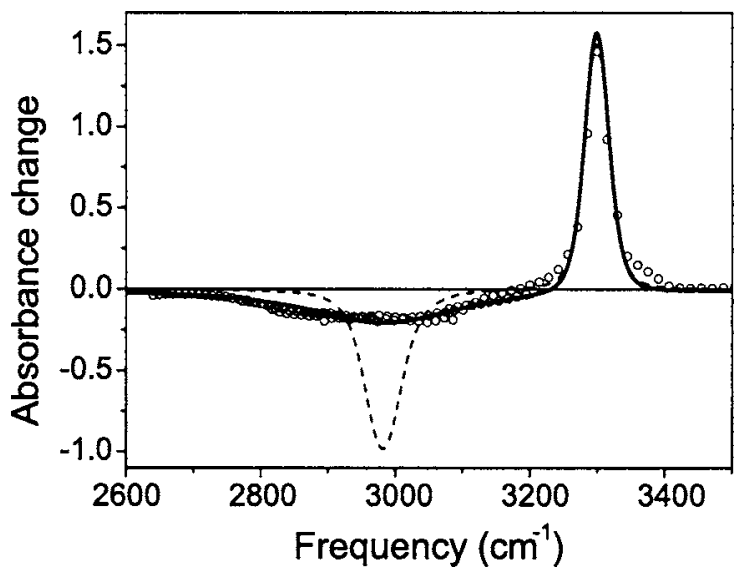

FIG. 9. Experimental $(\bigcirc)$ and calculated transient line shapes. Dashed line: calculated transient line shape when the vibrational lifetime of the $v_{\mathrm{OH}}=2$ state, $T_{1}^{v=2}=T_{1}^{v=1} / 2=210 \mathrm{fs}$ (as would be expected for an approximate harmonic oscillator). Solid line: Best fit between experiment and calculation, obtained by assuming a vibrational lifetime of the $v_{\mathrm{OH}}=2$ state of $T_{1}^{v=2}$ $=20 \mathrm{fs}$. 
We conclude that under the assumption that $T_{1}^{v=2}$ $=T_{1}^{v=1} / 2$, the LS model cannot give a proper description of the $v_{\mathrm{OH}}=1 \rightarrow 2$ absorption line shape. The LS model can only give rise to strong line broadening for this transition, when the $v_{\mathrm{OH}}=2$ energy is close to the top of the water autodissociation barrier. We find, however, that this is only the case for relatively short hydrogen bonds $(R \sim 2.65 \AA)$ that do not occur in ice $(R \sim 2.76 \pm 0.1 \AA)$.

We have tried to find different parameterizations of the LS potential [Eq. (5)] that would lower the top of the water autodissociation barrier, and bring it closer to the $v_{\mathrm{OH}}=2$ energy of longer hydrogen bonds. We found, however, that lowering the barrier unavoidably implies a strong increase of the anharmonicity of the $\mathrm{OH}$-stretch vibration. This results in $v_{\mathrm{OH}}=1 \rightarrow 2$ transition frequencies that are far too redshifted compared to the experimental observations. A low anharmonicity of the $\mathrm{OH}$-stretch potential of ice is also in line with the nearly harmonic value for the $\mathrm{OH}$-stretch overtone $\mathrm{ab}$ sorption $\left(6300 \mathrm{~cm}^{-1}\right){ }^{10}$

This leaves us with the remaining possibility that the broad $v_{\mathrm{OH}}=1 \rightarrow 2$ line shape is determined primarily by a very short lifetime of the $v_{\mathrm{OH}}=2$ vibrational state, i.e., $T_{1}^{v=2}$ $\ll T_{1}^{v=1} / 2$. We find good agreement between the experimental and calculated transient spectra when $T_{1}^{v=2} \approx T_{1}^{v=1} / 20$, making $T_{1}^{v=2}$ only $20 \pm 4 \mathrm{fs}$, as illustrated in Fig. 9. An explanation for this very short lifetime of the $v_{\mathrm{OH}}=2$ vibrational state can be found in the resonance of the $v_{\mathrm{OH}}=1 \rightarrow 2$ transition with the overtone of the bending vibration. This allows for very fast energy redistribution from the $v_{\mathrm{OH}}=2$ vibrational state.

\section{COMPARISON WITH LIQUID WATER}

We have shown that the relaxation of the $\mathrm{OH}$-stretch vibration of HDO: $\mathrm{D}_{2} \mathrm{O}$ ice proceeds via an intermediate state with a blueshifted absorption. A similar intermediate state is present in the vibrational relaxation pathway of liquid water. ${ }^{15}$ For liquid $\mathrm{H}_{2} \mathrm{O}$, the full spectrum of the intermediate state is hard to observe, because it is dominated by the pure pump-probe and heating signals $\left(\Delta \sigma_{\mathrm{PP}}\right.$ and $\Delta \alpha_{T}$, respectively). For HDO: $\mathrm{D}_{2} \mathrm{O}$ ice, the intermediate state absorption can be observed more clearly, because it appears spectrally separated, and because the heating effect is less pronounced.

We would like to comment on why proton delocalization can explain the broadening of the induced absorption in the case of liquid water, but not in the case of ice. This relates mainly to the very different hydrogen bond distributions that occur in ice and water. Ice is highly ordered and a hydrogen bond length spread of only $2.759-2.767 \AA$ is present. ${ }^{4}$ The liquid phase of water contains much more disorder, showing a spread of 2.6-2.9 $\AA^{17}$ Therefore, very short hydrogen bonds occur in water, for which the LS potential allows the proton to delocalize in the $v_{\mathrm{OH}}=2$ state. This qualitative difference in short and long hydrogen bonds makes the induced absorption of water very asymmetric. In the case of ice, the induced absorption line shape is determined by the lifetime, and its absorption is therefore very symmetric. For several reasons, the $v_{\mathrm{OH}}=1 \rightarrow 2$ transition linewidth in water is expected to be less dominated by lifetime broadening. The life- time of the $v_{\mathrm{OH}}=1$ state in water is much longer than in ice (740 fs). Therefore, it is likely that the lifetime of the $v_{\mathrm{OH}}$ $=2$ state in water will be longer as well. Most importantly, a longer $v_{\mathrm{OH}}=2$ lifetime for HDO: $\mathrm{D}_{2} \mathrm{O}$ water is expected than for HDO: $\mathrm{D}_{2} \mathrm{O}$ ice, since the $\mathrm{H}-\mathrm{O}-\mathrm{D}$ bending overtone is no longer in complete Fermi resonance with the $\mathrm{OH}$-stretch vibration.

It is remarkable that very slow ( $>100 \mathrm{ps})$ thermal dynamics is observed in ice but not in liquid water. The $\mathrm{OH}-$ stretch absorption in ice is much sharper than in water, which makes thermal signals (usually related to a small frequency shifts and cross section changes) relatively stronger. The amplitude of a signal resulting from a thermally induced frequency shift $\delta$ of a Gaussian band of width $\mu$ scales as $\delta / \mu^{2}$, thus quadratically decreasing with the linewidth. Because the water $\mathrm{OH}$-stretch absorption is four to five times broader than the ice $\mathrm{OH}$-stretch absorption, thermal effects are 20 times weaker (assuming similar thermally induced frequency shifts). Absorption lines in the liquid phase that are equally narrow as the $\mathrm{OH}$-stretch line in $\mathrm{HDO}: \mathrm{D}_{2} \mathrm{O}$ ice can also show long-time-scale thermal dynamics, ${ }^{22}$ similar to the slow dynamics reported here for ice.

\section{CONCLUSIONS}

We find that the vibrational relaxation of ice proceeds via an intermediate state, which has a blueshifted absorption by $50 \pm 20 \mathrm{~cm}^{-1}$. The intermediate likely corresponds to the $\mathrm{OH}$-stretch absorption of water molecules of which the $\mathrm{H}-\mathrm{O}-\mathrm{D}$ bending vibration is excited, as a result of vibrational relaxation. By fitting our data to a kinetic model, we can separate the pure pump-probe spectrum from the response of the intermediate state. We find the vibrational lifetime of ice to be $420 \pm 30 \mathrm{fs}$, in accordance with earlier studies, ${ }^{13}$ and the lifetime of the intermediate state to be $390 \pm 40$ fs.

We observe thermally induced absorption changes of the $\mathrm{OH}$-stretch mode that evolve on a time scale of tens to hundreds of picoseconds. These transmission changes likely relate to collective reorganizations of the ice lattice in response to heating.

A striking feature of the pure pump-probe spectrum of ice is the strongly broadened $v_{\mathrm{OH}}=1 \rightarrow 2$ transition. Such broadening was also observed for liquid water, and could be explained from proton delocalization in the $v_{\mathrm{OH}}=2$ state. ${ }^{11,17}$ We modeled the $v_{\mathrm{OH}}=0 \rightarrow 1$ and $v_{\mathrm{OH}}=1 \rightarrow 2$ line shapes of ice using the Lippincott-Schroeder model for the OH-stretch potential. Using this model, we find that proton delocalization cannot occur in the $v_{\mathrm{OH}}=2$ vibrational state of ice. Delocalization occurs only for fairly short (strong) hydrogen bonds that are not present in the ice crystal. Proton delocalization therefore does not contribute to the observed broadening of the induced absorption.

We find that the strong broadening of the induced absorption follows from a very short vibrational lifetime of the $v_{\mathrm{OH}}=2$ state $\left(T_{1}^{v=2}=20 \pm 4 \mathrm{fs}\right)$. This short lifetime can be explained from the resonance of the $v_{\mathrm{OH}}=1 \rightarrow 2$ transition with the overtone of the $\mathrm{H}-\mathrm{O}-\mathrm{D}$ bending vibration. 


\section{ACKNOWLEDGMENTS}

This work is part of the research program of the Stichting voor Fundamenteel Onderzoek der Materie (FOM), which is financially supported by the Nederlandse Organisatie voor Wetenschappelijk Onderzoek (NWO). We thank Pieter Rein ten Wolde for critically reading the manuscript. One of us (A.M.D.) wishes to thank Harm Geert Muller for useful discussions. Hinco Schoenmaker is gratefully acknowledged for technical support.

\section{APPENDIX A: KINETIC MODELING}

The following equations describe the dynamics of the molecules that were excited by the pump pulse:

$$
\begin{aligned}
& \frac{d N_{1}}{d t}=-\frac{1}{T_{1}} N_{1}, \\
& \frac{d N_{0}^{*}}{d t}=\frac{1}{T_{1}} N_{1}-\frac{1}{\tau_{\text {eq }}} N_{0}^{*}, \\
& \frac{d N_{0}}{d t}=\frac{1}{\tau_{\text {eq }}} N_{0}^{*} .
\end{aligned}
$$

$N_{1}, N_{0}^{*}$, and $N_{0}$ refer to the populations of the levels depicted in Fig. 4. As boundary conditions, we assume that on $t=0$ the number of molecules in the excited state equals the number of molecules removed from the ground state, $N_{0}(0)$ $=-N_{1}(0)$, and $N_{0}^{*}(0)=0$. We then find

$$
\begin{aligned}
& N_{1}(t)=N_{1}(0) e^{-t / T_{1}}, \\
& N_{0}^{*}(t)=N_{1}(0) \frac{\tau_{\mathrm{eq}}}{T_{1}-\tau_{\mathrm{eq}}}\left(e^{-t / T_{1}}-e^{-t / \tau_{\mathrm{eq}}} .\right.
\end{aligned}
$$

When the excited molecules relax to the ground state, the entire ice crystal gets gradually heated. Because of this heating, the OH-stretch cross-section spectrum $\left[\sigma_{01}(\omega, t)\right]$ slightly blueshifts, resulting in thermal absorption changes $\Delta \alpha_{T}(\omega, t)$ observed in the experiments. We assume the change of the spectrum $\sigma_{01}(\omega, t)$ upon heating is proportional to the total number of molecules $N_{\text {rel }}$ that have decayed to the ground state,

$$
\begin{aligned}
& \Delta \alpha_{T}(\omega, t) \propto N_{\text {rel }}(t) \\
& \quad=\int_{t^{\prime}=0}^{t} \frac{1}{\tau_{\mathrm{eq}}} N_{0}^{*}\left(t^{\prime}\right) d t^{\prime} \\
& \quad=N_{1}(0)-N_{1}(0)\left(\tau_{\mathrm{eq}} e^{-t / T_{\mathrm{eq}}}-T_{1} e^{-t / T_{1}}\right) /\left(\tau_{\mathrm{eq}}-T_{1}\right) .
\end{aligned}
$$

Heating affects the cross sections of all molecules, not only of the initially excited number of molecules $N_{1}(0)$, as we implicitly assume above. It can be shown that taking into account the heating effect on all molecules, in fact, gives the same expression for $\Delta \alpha_{T}$ as above if the fraction of excited molecules $N_{1}(0) / C$ is small, which is the case in our experiment. ${ }^{14}$

\section{APPENDIX B: DETAILS OF LINE SHAPE CALCULATION}

Assuming a classical fluctuating bath, the dipole time correlation function can be written as

$$
\begin{aligned}
\left\langle\tilde{\mu}_{i j}(0) \tilde{\mu}_{i j}(t)\right\rangle & =C_{i j}(t) \\
& =\left\langle u_{i j}(t) u_{i j}(0) m_{i j}(t) m_{i j}(0) e^{\left.-i \int_{0}^{\tau} \omega_{i j}(\tau) d \tau-\Gamma_{i j}{ }^{t}\right\rangle}\right.
\end{aligned}
$$

where we defined $\hat{\epsilon}$ as the laser field polarization, $\vec{M}=M \hat{M}$ as the molecular dipole moment, $m_{i j}=\langle i|M| j\rangle$ as the magnitude of the transition dipole moment, and $u(t)=\hat{\epsilon} \cdot \hat{M}$ as the molecular orientation. The $\Gamma_{i j}$ equal the dipole dephasing rates due to vibrational relaxation, which are given by

$$
\begin{aligned}
& \Gamma_{01}=\frac{1}{2 T_{1}^{v=1}}, \\
& \Gamma_{12}=\frac{1}{2 T_{1}^{v=1}}+\frac{1}{2 T_{1}^{v=2}} .
\end{aligned}
$$

$T_{1}^{v=j}$ equals the vibrational population decay time for vibrational state $|j\rangle$ (we write $2 T_{1}$ since within the density matrix formulism, polarizations are proportional to coherences, which dephase due to vibrational relaxation at half the population relaxation rate of the corresponding excited state ${ }^{23}$ ).

For ice we can neglect the effects of reorientation, and take $u_{i j}(t)=1$. In our model, the dipole transition moments $m_{i j}$ and frequencies $\omega_{i j}$ depend parametrically on the hydrogen bond length $\mathrm{R}$. Length modulations in $\mathrm{R}$ will result in time-dependent fluctuations of $\omega_{i j}$ and $m_{i j}$. We assume that the OH-stretch motion depends parametrically on the hydrogen bond stretch $(\mathrm{O} \cdots \mathrm{O})$ coordinate. This type of adiabatic approach is commonly used in describing the coupling between a high-frequency $\mathrm{O}-\mathrm{H}$ stretch vibration and a lowfrequency $\mathrm{O}-\mathrm{H} \cdots \mathrm{O}$ hydrogen bond. The approximation will be valid when the $\mathrm{O} \cdots \mathrm{O}$ modulations are slow compared to the $\mathrm{OH}$-stretch motion, which is a fairly reasonable assumption since the thermally excited phonon vibrations $\left(\sim 200 \mathrm{~cm}^{-1}\right)$ in ice are an order of magnitude slower in frequency than the $\mathrm{OH}$-stretch vibrations $\left(\sim 3000 \mathrm{~cm}^{-1}\right) .{ }^{24} \mathrm{We}$ solve the one-dimensional Schrödinger Equation for the LS potential with a parametric dependence on the hydrogen bond coordinate $R$ using a Numerov algorithm ${ }^{25}$ [we adopt reduced units of energy $\left(\mathrm{cm}^{-1}\right)$, length $(\AA)$, and mass $\left(\mathrm{m} / \hbar^{2}\right)$, and take the reduced mass of an $\mathrm{OH}$ oscillator equal to $\left.0.0279 \AA^{-2} / \mathrm{cm}^{-1}\right]$. This results in eigenenergies $E_{v}$ and eigenfunctions $|v\rangle$ up to $v_{\mathrm{OH}}=2$ for each hydrogen bond coordinate $R$. The $\mathrm{OH}$-stretch absorption frequency is given by the relation $\omega_{i j}(R)=\left(E_{j}-E_{i}\right) / \hbar$ and the absorption cross sections $m_{i j}$ are found by calculating the matrix elements $\langle i|r| j\rangle$ for each $v_{\mathrm{OH}}=i \rightarrow j$ transition.

Making explicit the ensemble average over the hydrogen bond length distributions in Eq. (6), we have

$$
C_{i j}(t)=\int_{0}^{\infty} d R^{\prime} \cdot f_{\text {stat }}\left(R^{\prime}\right) \int_{0}^{\infty} d R \cdot C_{i j}\left(R, R^{\prime}, t\right),
$$

where 


$$
C_{i j}\left(R, R^{\prime}, t\right)=P_{i j}\left(R, R^{\prime}, 0\right) P_{i j}\left(R, R^{\prime}, t\right)
$$

$P_{i j}\left(R, R^{\prime}, t\right)$ now equals a macroscopic time-dependent polarization. The $\left(R, R^{\prime}\right)$ hydrogen bond coordinates refer to the dynamic and static hydrogen bond distributions, as discussed in Sec. IV B, i.e., $P_{i j}\left(R, R^{\prime}, t\right)$ is generated by the subset of oscillators that experience an instantaneous hydrogen bond length $R$ and have an equilibrium hydrogen bond length $R^{\prime}$. $P_{i j}\left(R, R^{\prime}, t\right)$ accumulates phase by a factor of $e^{i \omega(R) t}$, but gains additional phase and changes amplitude as a result of the frequency modulations. The line shape calculation is now reduced to the calculation of the dipole time correlation functions $C_{i j}\left(R, R^{\prime}, t\right)$.

The length spread and dynamics of the hydrogen bonds in the ice crystal were discussed in Sec. IV B. These are related to a frequency spread and modulation in $\mathrm{OH}$-stretch frequencies by the LS model. The inhomogeneous spread in hydrogen bonds $f_{\text {stat }}\left(R^{\prime}\right)$ follows a Gaussian distribution of width $\Delta_{R, \text { stat }}=0.4 \mathrm{pm}$ and mean $R_{0}=2.76 \AA$. Around each hydrogen bond length $R^{\prime}$, we center a distribution of hydrogen bond lengths $f_{\text {dyn }}\left(R, R^{\prime}\right)$ to account for the stochastic modulation of the hydrogen bonds by phonons. $f_{\text {dyn }}\left(R, R^{\prime}\right)$ equals a Gaussian distribution of width $\Delta_{R, \mathrm{dyn}}=1.0 \mathrm{pm}$ and mean $R^{\prime}$.

We rewrite the integrals in Eq. (B4) as discrete summations over the variables $\left(R, R^{\prime}\right)$, adopting integration steps of length $d R=1 \times 10^{-13} \mathrm{~m}$.

$$
C_{i j}(t)=\sum_{k} f_{\text {stat }}\left(R_{k}^{\prime}\right) \sum_{l} C_{i j}\left(R_{l}, R_{k}^{\prime}, t\right) .
$$

We assume that the pump and probe pulses are short compared to the system dynamics (impulsive pump-probe limit), and take the excited state and ground state dynamics fully uncorrelated and in thermal equilibrium. We can then neglect cross correlations between the different states in the third order response. We calculate the transient spectrum from the linear response for thermal equilibrium populations in both the ground and excited state.

For each transition $v_{\mathrm{OH}}=v-1 \rightarrow v$, each bin $l$ has an associated cross section $m_{l, v}$ as calculated from the LS model (this bin-associated transition dipole moment should not be confused with the earlier definition of the transition dipole moment $m_{i j}$, where the indices refer to vibrational states). The bins exchange population from bin $l=m$ to $l=n$ on a rate $k_{m, n}$ according to a diffusional process, to account for the stochastic stretching modulations of the hydrogen bond length. Assuming constant diffusion over the entire distribution of hydrogen bonds $\left[\left(k_{m, m+1}+k_{m+1, m}\right) / 2=k_{0}\right]$, and using detailed balance $k_{m, m+1} \cdot f_{\mathrm{dyn}}\left(R_{m}, R_{k}^{\prime}\right)=k_{m+1, m} \cdot f_{\mathrm{dyn}}\left(R_{m+1}, R_{k}^{\prime}\right)$, the hop rates are given by

$$
\begin{aligned}
k_{m, n}= & k_{0} \frac{2 f_{\mathrm{dyn}}\left(R_{n}, R_{k}^{\prime}\right)}{f_{\mathrm{dyn}}\left(R_{m}, R_{k}^{\prime}\right)+f_{\mathrm{dyn}}\left(R_{n}, R_{k}^{\prime}\right)}, \\
& \text { for } n=m \pm 1 ; \quad 0, \quad \text { for } n \neq m \pm 1,
\end{aligned}
$$

$$
k_{0}=\frac{D_{\mathrm{hop}}}{d R^{2}}
$$

where $D_{\text {hop }}$ is the diffusion constant determining the rate of the stochastic hydrogen bond modulations.

For simplicity, we will write the simple indices $(l, k)$ for the discretized variables $\left(R_{l}, R_{k}^{\prime}\right)$ and index $v$ to denote the $v_{\mathrm{OH}}=v-1 \rightarrow v$ transition. In each time step of the integration we keep track of the complex amplitude $P_{l, k, v}(t)$ $\equiv P_{v-1 v}\left(R_{l}, R_{k}^{\prime}, t\right)$ of the polarization associated with each bin $l$, according to

$$
\begin{aligned}
\frac{d P_{l, k, v}}{d t}= & \left(i \omega_{l}-\Gamma_{v-1 v}-k_{l, l+1}-k_{l, l-1}\right) P_{l, k, v} \\
& +k_{l+1, l} \frac{m_{l, v}}{m_{l+1, v}} P_{l+1, k, v}+k_{l-1, l} \frac{m_{l, v}}{m_{l-1, v}} P_{l-1, k, v}, \\
P_{l, k, v}(0) & =f_{\mathrm{dyn}}\left(R_{l}, R_{k}^{\prime}\right) m_{l, v} .
\end{aligned}
$$

$\Gamma_{v-1 v}$ equals the polarization decay due to vibrational relaxation [see Eq. (B2) and Eq. (B3)]. Equation (B9) consists of a system of $l$ linear differential equations for each $v_{\mathrm{OH}}=v$ $-1 \rightarrow v$ transition and each hydrogen bond coordinate $R_{k}^{\prime}$. This system is solved by numerical integration. The only remaining fit parameter is $D_{\text {hop }}$, which was adjusted to give the best description of the linear $v_{\mathrm{OH}}=0 \rightarrow 1$ absorption $\left(D_{\text {hop }}=30 \mathrm{pm}^{2} / \mathrm{ps}\right)$

In the limit of a Gaussian hydrogen bond length distribution, a linear mapping between $R$ and $\mathrm{OH}$-stretch frequency, and a white cross-section spectrum, $D_{\text {hop }}$ is related to the frequency-frequency correlation time $\tau_{c}$ by ${ }^{26,27}$

$$
\begin{aligned}
\tau_{c} & =\frac{d R^{2}}{D_{\text {hop }}}\left(1-\sqrt{1-2 d R^{2} / \Delta_{R, \text { dyn }}^{2}}\right)^{-1} \\
& \rightarrow \frac{\Delta_{R, \text { dyn }}^{2}}{D_{\text {hop }}}, \quad \text { if } d R \ll \Delta_{R, \text { dyn }} .
\end{aligned}
$$

Because of the anharmonicities included in the LS model, the mapping between hydrogen bond coordinates $R$ and the $\mathrm{OH}$-stretch frequencies and cross sections is, however, a more complicated relation (including non-Condon effects). Numerical calculation of $\tau_{c}$ by determining the exponential decay time of the frequency-frequency correlation function $\langle\delta \omega(0) \delta \omega(t)\rangle\left[\delta \omega(t)=\omega(t)-\omega_{0}, \omega_{0}\right.$ being the mean frequency of the distribution], resulted in $\tau_{c}=0.33 \mathrm{ps}$ for the $v_{\mathrm{OH}}=0$ $\rightarrow 1$ transition and $\tau_{c}=0.27 \mathrm{ps}$ for the $v_{\mathrm{OH}}=1 \rightarrow 2$ transition. From Eq. (B11) we find $\tau_{c} \simeq 0.3 \mathrm{ps,}$, which shows that the distributions are near Gaussian.

\footnotetext{
${ }^{1}$ L. Pauling, J. Am. Chem. Soc. 57, 2680 (1935).

${ }^{2}$ W. Kuhs and M. Lehmann, J. Phys. (Paris) 48, 3 (1987).

${ }^{3}$ K. Rottger, A. Endriss, J. Ihringer, S. Doyle, and W. F. Kuhs, Acta Crystallogr., Sect. B: Struct. Sci. 50, 644 (1994).

${ }^{4}$ J.-L. Kuo, M. L. Klein, and W. F. Kuhs, J. Chem. Phys. 123, 134505 (2005).

5 J. E. Bertie and E. Whalley, J. Chem. Phys. 40, 1637 (1963).

${ }^{6}$ G. Seifert, K. Weidlich, and H. Graener, Phys. Rev. B 56, 231 (1997).

${ }^{7}$ V. Buch and J. Devlin, J. Chem. Phys. 110, 3437 (1999).

${ }^{8}$ M. Wojcik and V. Buch, J. Chem. Phys. 99, 2332 (1993).

${ }^{9}$ E. R. Lippincott and R. Schroeder, J. Chem. Phys. 23, 1099 (1955).

${ }^{10}$ C. Haas and D. F. Hornig, J. Chem. Phys. 32, 1763 (1960).
} 
${ }^{11}$ H. J. Bakker and H.-K. Nienhuys, Science 297, 587 (2002).

${ }^{12}$ A. M. Dokter, S. Woutersen, and H. J. Bakker, Proc. Natl. Acad. Sci. U.S.A. 103, 15355 (2006).

${ }^{13}$ S. Woutersen, U. Emmerichs, H. K. Nienhuys, and H. J. Bakker, Phys. Rev. Lett. 81, 1106 (1998).

${ }^{14}$ Y. L. A. Rezus and H. J. Bakker, J. Chem. Phys. 123, 114502 (2005).

${ }^{15}$ J. Lindner, P. Vöhringer, M. S. Pshenichnikov, D. Cringus, D. A. Wiersma, and M. Mostovoy, Chem. Phys. Lett. 421, 329 (2006).

${ }^{16}$ H. Iglev, M. Schmeisser, K. Simeonidis, A. Thaller, and A. Laubereau, Nature (London) 439, 183 (2006).

${ }^{17}$ H. J. Bakker, H.-K. Nienhuys, G. Gallot, N. Lascoux, G. M. Gale, J.-C. Leicknam, and S. Bratos, J. Chem. Phys. 116, 2592 (2002).

${ }^{18}$ F. Sciortino and G. Corongiu, J. Chem. Phys. 98, 5694 (1993).

${ }^{19}$ M. Falk, J. Chem. Phys. 87, 28 (1987).
${ }^{20}$ J. Braun, A. Glebov, A. P. Graham, A. Menzel, and J. P. Toennies, Phys. Rev. Lett. 80, 2638 (1998).

${ }^{21}$ R. Kubo, M. Toda, and N. Hashitsume, Statistical Physics II: Nonequilibrium Statistical Mechanics, 2nd ed. (Springer-Verlag, Berlin, 1995).

${ }^{22}$ V. Botan, E. H. G. Backus, R. Pfister, A. Moretto, M. Crisma, C. Toniolo, P. H. Nguyen, G. Stock, and P. Hamm, Proc. Natl. Acad. Sci. U.S.A. 104, 12749 (2007).

${ }^{23}$ R. W. Boyd, Nonlinear Optics (Academic, San Diego, 1992), p. 122.

${ }^{24}$ C. J. Burnham, J. Li, S. S. Xantheas, and M. Leslie, J. Chem. Phys. 110, 4566 (1999).

${ }^{25}$ S. Koonin, Computational Physics (Benjamin, Menlo Park, CA, 1986).

${ }^{26}$ A. I. Burshtein and V. S. Malinovsky, J. Opt. Soc. Am. B 8, 1098 (1991).

${ }^{27}$ H. Bakker, S. Woutersen, and H.-K. Nienhuys, Chem. Phys. 258, 233 (2000). 